\title{
Assessing the risk of rapid fibroid growth in patients with asymptomatic solitary uterine myoma using a multivariate prediction model
}

\author{
Qingxiu Li ${ }^{1}$, Jiehui Zhong ${ }^{2}$, Dongyi Yi ${ }^{3}$, Genqiang Deng ${ }^{3}$, Zezhen Liu ${ }^{2}$, Wei Wang ${ }^{4}$ \\ ${ }^{1}$ Health Management Medical Center, The First Affiliated Hospital of Guangzhou Medical University, Guangzhou, China; ${ }^{2}$ Department of Urology, \\ Minimally Invasive Surgery Center, Guangdong Key Laboratory of Urology, Guangzhou Urology Research Institute, The First Affiliated Hospital \\ of Guangzhou Medical University, Guangzhou, China; ${ }^{3}$ Network and Information Technology Department, Huazhong University of Science and \\ Technology Union Shenzhen Hospital, Shenzhen, China; ${ }^{4}$ Department of Obstetrics and Gynecology, The First Affiliated Hospital of Guangzhou \\ Medical University, Guangzhou, China \\ Contributions: (I) Conception and design: Q Li, Z Liu; (II) Administrative support: J Zhong, Z Liu; (III) Provision of study materials or patients: \\ G Deng, D Yi; (IV) Collection and assembly of data: G Deng, D Yi; (V) Data analysis and interpretation: W Wang; (VI) Manuscript writing: All \\ authors; (VII) Final approval of manuscript: All authors. \\ Correspondence to: Gengqiang Deng. Network and Information Technology Department, Huazhong University of Science and Technology Union \\ Shenzhen Hospital, Shenzhen, China. Email: denggenqiang@gmail.com; Zezhen Liu. Department of Urology, Minimally Invasive Surgery \\ Center, Guangdong Key Laboratory of Urology, Guangzhou Urology Research Institute, The First Affiliated Hospital of Guangzhou Medical \\ University, Kangda Road 1\#, Haizhu District, Guangzhou 510230, China. Email: liuzz2016@qq.com; Wei Wang. Department of Obstetrics and \\ Gynecology, The First Affiliated Hospital of Guangzhou Medical University, Yanjiang Road 151\#, Yuexiu District, Guangzhou 510230, China. \\ Email: smugowwang@126.com.
}

Background: Long-term conservative approaches are effective management options for asymptomatic uterine fibroids, but not for uterine myomas with excessive growth. In this investigation, a regression model was constructed to evaluate the clinical characteristics related to uterine fibroids' growth.

Methods: In this retrospective study, 19,840 patients with ultrasound-diagnosed uterine fibroids were identified from six centers between 2013 and 2019. In total, 739 patients were followed up for more than 1 year with B-ultrasound test results and clinical test results and had no acute events or surgical treatments. The endpoint was changed in the size of the uterine fibroids. Multivariate stepwise logistic regression analysis was used to identify predictors of uterine fibroid growth, and these were used to build a prediction model. The prediction model's discrimination, calibration, and clinical efficacy were assessed using the area under the curve (AUC)/index of concordance (C-index), calibration plot, decision curve analysis, and clinical impact curve. Internal validation was performed using bootstrapping validation. A linear regression model was constructed to predict uterine fibroids' growth rate without the occurrence of acute events.

Results: A total of 513 patients presented with significant growth of uterine fibroids, with an average follow-up time of 927 days, and 267 patients showed negative growth, with an average follow-up time of 960 days. Age, follicle-stimulating hormone (FSH), low-density lipoprotein (LDL), luteinizing hormone (LH), total cholesterol (TCHO), and neutrophil to lymphocyte ratio (NLR) were the main influential factors that predicted the uterine fibroid growth state, and these were used to develop a nomogram with predictive accuracy (AUC: 0.825). A linear regression prediction model was built based on the following factors: FSH, high-density lipoprotein (HDL), LH, triglyceride (TRIG), TCHO, and lymphocyte to monocyte ratio (LMR). The mean square error (MSE) was 0.32 .

Conclusions: This study directly measured the growth rate of uterine fibroids. A prediction model assessing the growth rate of asymptomatic uterine fibroids was established. This model is useful for the early detection of potentially rapidly growing uterine fibroids in patients.

Keywords: Single asymptomatic uterine fibroid; growth ratio; linear regression prediction model 
Submitted Jun 09, 2020. Accepted for publication Dec 06, 2020.

doi: 10.21037/atm-20-4559

View this article at: http://dx.doi.org/10.21037/atm-20-4559

\section{Introduction}

Uterine fibroids are benign neoplasms of the uterus. It is more common in women between the ages of 30 to 50 years (1). In China, more than $20 \%$ of women over the age of 35 have uterine fibroids. The prevalence of uterine fibroids is about $11.21 \%$, and the rate of malignant change is approximately $0.30 \%$ (2). In many patients, uterine fibroids are discovered during physical examination. Most of these uterine fibroids do not cause noticeable symptoms in the short term. Some uterine fibroids grow slowly or even stay unchanged for long periods, and these patients failed to seek early treatment or do not receive any treatment. Another subset of uterine fibroids can grow rapidly and trigger acute events within a relatively short period, such as anemia, pelvic pain, increased urinary frequency, and abdominal distension. The annual treatment costs of uterine fibroids are estimated at \$6-34 billion in the United States, with similar high costs internationally (3).

The growth of uterine fibroids is affected by estrogen, luteinizing hormone (LH), and follicle-stimulating hormone (FSH) (4-7). High levels of hormones stimulate the rapid growth of fibroids in the uterus (8). Interestingly, uterine fibroids in postmenopausal women appear to shrink (9). Lipid metabolism in the body can also affect uterine fibroids (10). One Japanese case-control study found that the percent of body fat, but not the body mass index (BMI), is associated with a greater incidence of uterine fibroids (11). However, another study revealed that oral contraceptives were a risk factor for the incidence of uterine leiomyoma in Chinese women of childbearing age. The innate immune status also affected the growth of uterine fibroids (12). Naturally, there are still many unknown factors that affect the growth of uterine fibroids. This retrospective study analyzed patients' clinical characteristics with uterine fibroids and examined the growth ratio of uterine fibroids using a multivariate prediction model.

Clinical prediction models have become increasingly popular over recent years (13). As a quantitative tool for assessing risks and benefits, clinical prediction models can provide more objective and accurate information to aid doctors, patients, and health administrators (14). However, to date, there is currently no clinical model for predicting the growth of uterine fibroids. This study aimed to establish a predictive model to assist clinicians in assessing the growth of uterine fibroids. Our prediction model could identify patients with rapidly growing uterine fibroids from a cohort of singlefibroid asymptomatic patients. We present the following article in accordance with the TRIPOD reporting checklist (available at http://dx.doi.org/10.21037/atm-20-4559).

\section{Methods}

The study was conducted in accordance with the Declaration of Helsinki (revised in 2013) and was approved by the institutional research ethics committee of Huazhong University of Science and Technology Union Shenzhen Hospital. All accessed data were anonymized (ID: LW2020-007). Due to the retrospective nature of the research, the requirement for patient informed consent was waived.

\section{Study population}

This was a retrospective multicenter study conducted at the following 6 institutions: The First Affiliated Hospital of Guangzhou Medical University, Huazhong University of Science and Technology Union Shenzhen Hospital, Shenzhen Shekou People's Hospital, Shenzhen Nanshan Maternity and Child Healthcare Hospital, Southern University of Science and Technology Hospital, and Shenzhen Nanshan District Institute for Chronic Diseases Prevention and Treatment. The follow-up period was from 2013 to 2019.

Patients with a B-ultrasound diagnosis of uterine fibroids with no associated symptoms (such as pain), and 3 or more follow-up consultations over 1 year or more, were included in this study. The exclusion criteria included patients who had an acute event during the follow-up that required surgical treatment, FSH concentrations greater than $40 \mathrm{U} / \mathrm{L}$; patients with incomplete clinical testing information in their followup data; and patients diagnosed with pregnancy or fatal diseases, such as cancer, during their follow-up period.

\section{Data collection}

Basic patient clinical data, including ultrasound results 
Table 1 The clinical characteristics of the 20,088 patients diagnosed with uterine fibroids by ultrasound scan

\begin{tabular}{lc}
\hline Variable & Value \\
\hline Age (year), mean \pm SD & $42.92 \pm 7.72$ \\
Size $\left(\mathrm{mm}^{2}\right)$, mean \pm SD & $933.20 \pm 1,529.79$ \\
Location-1, N (\%) & \\
Subserous myoma & $14,596(27.04)$ \\
Intramural myoma & $4,779(71.15)$ \\
Submucosal myoma & $213(0.97)$ \\
Location-2*, N (\%) & \\
Cervix & $162(0.73)$ \\
Uterus & $1,655(8.08)$ \\
Body of uterus & $14,339(70.53)$ \\
\hline
\end{tabular}

*, some patients are missing information. SD, standard deviation.

and hematological tests, were collected. Basic clinical characteristics included ID that was used to queried multiple hospitalization information, age, and marital status were collated. The longest diameter and widest diameter of the uterine fibroid was collected from the ultrasound report. Location data of the fibroids were collected from ultrasound reports. Location-1 was defined as uterine fibroids detected in the intramural, subserosal, and submucosal regions. Location-2 was defined as uterine fibroids detected in the bottom, cervix, and body of the uterus. The uterine fibroid size was measured as the product of the longest diameter by the widest diameter. The patient's age at the first follow-up and the last follow-up was documented. The average growth rate of uterine fibroids was measured as the change in size $\left(\mathrm{mm}^{2}\right)$ in uterine fibroids during the followup period divided by the duration of the follow-up period (days). Hematological tests included levels of creatinine (Cr, $\mu \mathrm{mol} / \mathrm{L}$ ), eosinophil (EOS, $\left.10^{9} / \mathrm{L}\right), \mathrm{FSH}(\mathrm{mIU} / \mathrm{mL})$, HDL (mmol/L), LDL (low-density lipoprotein, $\mathrm{mmol} / \mathrm{L}), \mathrm{LH}$ $(\mathrm{mU} / \mathrm{mL})$, lymphocytes $\left(10^{9} / \mathrm{L}\right)$, monocytes (Monc, $10^{9} / \mathrm{L}$ ), neutrophils (Neut, $\left.10^{9} / \mathrm{L}\right)$, serum albumin $(\mathrm{g} / \mathrm{L})$, total cholesterol (TCHO, mmol/L), triglycerides (TRIG, $\mathrm{mmol} / \mathrm{L}$ ), lymphocyte to monocyte ratio (LMR), monocyte to neutrophil ratio (MNR), neutrophil to lymphocyte ratio (NLR), high-density lipoprotein to low-density lipoprotein ratio (HLR), and estradiol (E2, pmol/L).

\section{Statistical analysis}

Statistical analysis was performed using $\mathrm{R}$ software (version 3.6.3; http://www.R-project.org). Two-sided $\mathrm{P}<0.05$ was regarded as statistically significant.

The classification of individual cases with positive growth was based on the growth ratio of uterine fibroids larger than $2.5 \mathrm{~mm}^{2} / \mathrm{d}$. Since B-ultrasound measurement of uterine fibroids is not very accurate, a growth rate greater than $2.5 \mathrm{~mm}^{2} / \mathrm{d}$ was defined as significant growth. The patient characteristics were summarized using counts for categorical data and the mean for continuous variables. Multivariate logistic regression was used to explore the clinical and imaging characteristics against uterine fibroid growth. Reduced model selection was performed using a backward step-down selection process. The area under the curve (AUC) of the receiver operating characteristic (ROC) curve was used to quantify the prediction accuracy. A nomogram was developed to predict uterine fibroid growth using the regression coefficients of each predictor. In this process, each predictor's impact on uterine fibroid growth was weighted and converted to a numerical score. The individual numerical scores corresponding to each predictor were added to determine the total score. It was then translated to provide the predicted chance of uterine fibroid growth using a nomogram. The calibration curves, the C-index, decision curve, and clinical impact curve were used to evaluate the nomogram (15-17). A linear regression model was developed to predict uterine fibroids' growth rate by analyzing patients with an average growth rate greater than $2.5 \mathrm{~mm}^{2} /$ day.

\section{Results}

\section{Patient characteristics}

The clinical characteristics of the 19,840 patients diagnosed with uterine fibroids by ultrasound are shown in Table 1 . The relationship between the number of cases and age is shown in Figure 1. Between 19 and 46 years of age, the number of cases increased with age. After 46 years of age, the number of cases decreased significantly with age. The size of the uterine fibroids was less than $5,000 \mathrm{~mm}^{2}$ in $97.35 \%$ of the patients. Following the inclusion and exclusion criteria, a total of 739 uterine fibroid patients were included in the study. The clinical characteristics 


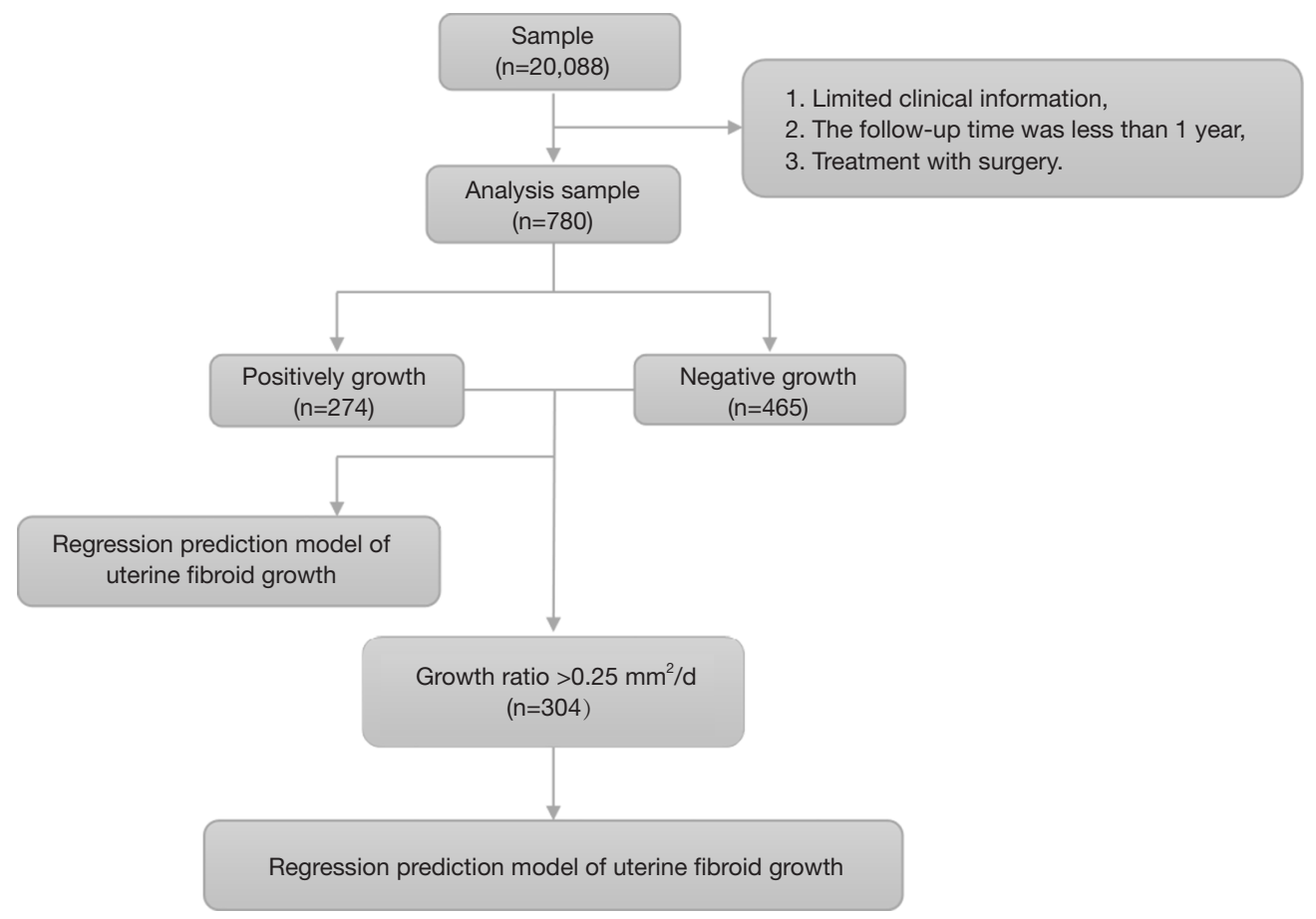

Figure 1 The relationship between the number of cases and the age of the patients.

of these patients are shown in Table 2. The mean (range) patient follow-up time was $948(367-2,080)$ days. The location characteristics of the 739 patients diagnosed with uterine fibroids by ultrasound scan are shown in Table 3 . The average growth rate of uterine fibroids in the negative growth group and the positive growth group was -0.43 and $0.98 \mathrm{~mm}^{2} / \mathrm{d}$. Clinical factors significantly different in the 2 groups included age, FSH, LH, neutrophils, and NLR.

\section{A predictive model for the growth direction of uterine fibroids}

The endpoint event is a fibroid growth rate greater than $2.5 \mathrm{~mm}^{2} / \mathrm{d}$. Table 4 shows the individual and joint predictive influence of various characteristics based on univariate and multivariate logistic regression. The following factors were associated with uterine fibroid growth: age, FSH, LDL, LH, TCHO, and NLR (Figure S1).

Stepwise multivariate logistic regression analysis identified six significant predictors for uterine fibroid growth in the study cohort, including age, FSH, LDL, LH, TCHO, and NLR (Table 5). A nomogram was developed using these six significant predictors to predict significant uterine fibroid growth (Figure 2). The sum of the corresponding scores in the six categories was calculated. An interpretation scale was built into the nomogram to assist physicians with translating the scores into the percent chance of positive growth. The total score reflects the chance of significant uterine fibroid growth. Patients with a high total score had a greater chance of significant uterine fibroid growth, while those with a low total score had a reduced chance of significant fibroid growth.

\section{Evaluation of the predictive model for uterine fibroid growth}

Figure $3 A$ shows the ROC curve of the logistics model in the test data. The AUC was 0.8235 , and the nomogram calibration plot demonstrated high reliability. The uterine fibroid growth was predicted using the model and the calibrated observations. The model showed the best fit when the uterine fibroids' growth ratio was between $0 \%$ and $50 \%$. When the uterine fibroids' growth ratio ranged from $50 \%$ to $70 \%$, the model overestimated the growth rate of uterine fibroids. When the predicted probability was greater than $70 \%$, the model underestimated uterine fibroids' growth rate (Figure $3 B$ ). The C-index for the prediction nomogram was 0.825 for the cohort, indicating that the model showed 
Table 2 The clinical characteristics of the 739 patients diagnosed with uterine fibroids by ultrasound scan

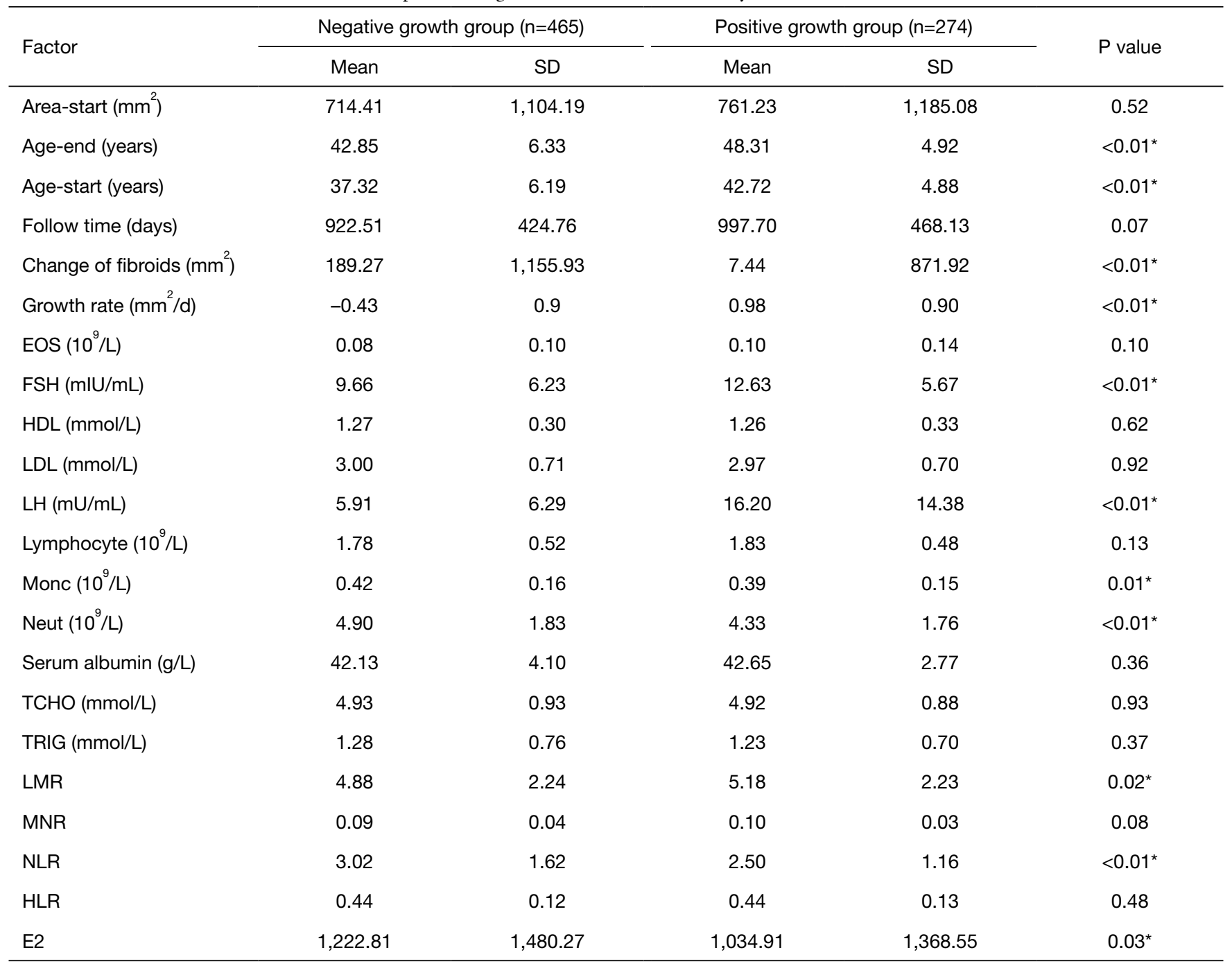

${ }^{*} \mathrm{P}<0.05$. SD, standard deviation. EOS, eosinophil; FSH, follicle-stimulating hormone; HDL, high-density lipoprotein; LDL, low-density lipoprotein; LH, luteinizing hormone; Monc, monocytes; Neut, neutrophils; TCHO, total cholesterol; TRIG, triglyceride; LMR, lymphocyte to monocyte ratio; MNR, monocytes to neutrophil ratio; NLR, neutrophil to lymphocyte ratio; HLR, high-density lipoprotein to low-density lipoprotein ratio; E2, estradiol.

good discrimination. As shown in the decision curve, the predictions based on the nomogram were clinically useful if the threshold values in the approximate range of 0.1 to 0.9 were used to determine the treatment strategy. Additionally, the decision analysis curve showed that predicting patients with fast-growing uterine fibroids with a risk threshold of 0.1 to 0.9 potentially yielded a net clinical benefit (Figure 3C). The clinical impact curve shows the estimated numbers of patients deemed high risk and true positives in the range of 0 to 0.4 using our risk model (Figure 3D).

\section{Linear regression prediction model for the uterine fibroid growth ratio}

A linear regression model was developed to predict uterine fibroids' growth rate in a population with significant growth (fibroid growth rate $>2.5 \mathrm{~mm}^{2} / \mathrm{d}$ ). In this cohort, $70 \%$ of patients were randomly selected as the training data, and the remaining $30 \%$ of patients represented the test data. By analyzing the information of the training data, a linear regression model was established (Table 6). The linear 
Table 3 The location characteristics of the 739 patients diagnosed with uterine fibroids by ultrasound scan

\begin{tabular}{lccc}
\hline & $\begin{array}{c}\text { Negative } \\
\text { growth group }\end{array}$ & $\begin{array}{c}\text { Positively } \\
\text { growth group }\end{array}$ & P value \\
\hline Location-1 (related to myometrium) & 183 & 0.32 \\
Intramural & 311 & 89 & \\
Subserosal & 144 & 2 & \\
Submucosal & 10 & & \\
Location-2 (myoma location) & & 19 & \\
Uterine bottom & 36 & 1 & \\
Cervix & 3 & 196 & \\
Body of uterus & 324 & 58 & \\
Unknown & 102 & 19 & \\
Marital status & & 243 & \\
Unmarried & 38 & 12 & \\
Married & 406 & 21 & \\
Remarriage & & &
\end{tabular}

Area-start is the size of the uterine fibroids measured by ultrasound at the initial follow-up. Age-start is the age at the initial follow-up. Age-end is the age at the last follow-up. The ratio is the change in fibroids/follow-up time $\left(\mathrm{mm}^{2} / \mathrm{d}\right)$. The max-area is the maximum area of myoma during the follow-up. Myoma related to the myometrium is classified as location-1. Myoma, located in the uterus, is defined as location-2 (Table 3). regression prediction model was used to predict the test data. The mean squared error (MSE) was 0.32 , and the $\mathrm{R}$-square value (coefficient of determination) was 0.79 . The predicted values were distributed around actual values (Figure 4). The error of the linear regression prediction model was normally distributed (Figure 5).

\section{Discussion}

The incidence rate of uterine fibroids is approximately $30 \%$ in women aged $30-50$ years. Most women with uterine

Table 5 Logistic regression analysis identified six significant predictors for uterine fibroid growth

\begin{tabular}{lc}
\hline Factor & Regression coefficient \\
\hline Age & 0.12 \\
FSH & 0.05 \\
LDL & -0.56 \\
LH & 0.09 \\
TCHO & 0.31 \\
NLR & -0.07 \\
Intercept & -6.46 \\
\hline
\end{tabular}

$\mathrm{FSH}$, follicle-stimulating hormone; LDL, low-density lipoprotein; $\mathrm{LH}$, luteinizing hormone; TCHO, total cholesterol; NLR, neutrophil to lymphocyte ratio.

Table 4 Univariate and multivariate logistic regression analysis of the predictors of significant fibroid growth

\begin{tabular}{|c|c|c|c|c|c|c|}
\hline \multirow{2}{*}{ Predictors } & \multicolumn{2}{|c|}{ Univariate unadjusted } & \multicolumn{4}{|c|}{ Multivariate adjusted } \\
\hline & OR $(95 \% \mathrm{Cl})$ & $\mathrm{P}$ & OR $(95 \% \mathrm{Cl})$ & $\mathrm{P}$ & OR $(95 \% \mathrm{Cl})$ & $\mathrm{P}$ \\
\hline Age & $1.189(1.152 \pm 1.231)$ & $<0.001$ & $1.132(0.964 \pm 1.331)$ & 0.131 & $1.151(1.112 \pm 1.195)$ & $<0.001$ \\
\hline $\mathrm{FSH}$ & $1.081(1.055 \pm 1.11)$ & $<0.001$ & $1.053(1.019 \pm 1.089)$ & 0.002 & $1.059(1.027 \pm 1.093)$ & $<0.001$ \\
\hline $\mathrm{LH}$ & $1.128(1.102 \pm 1.157)$ & $<0.001$ & $1.104(1.076 \pm 1.137)$ & $<0.001$ & $1.095(1.069 \pm 1.124)$ & $<0.001$ \\
\hline $\mathrm{TCHO}$ & $0.992(0.841 \pm 1.168)$ & 0.927 & $1.185(0.662 \pm 2.100)$ & 0.563 & $1.195(0.764 \pm 1.859)$ & 0.432 \\
\hline NLR & $0.757(0.666 \pm 0.852)$ & $<0.001$ & $0.570(0.336 \pm 0.891)$ & 0.022 & $0.864(0.749 \pm 0.990)$ & 0.039 \\
\hline
\end{tabular}

FSH, follicle-stimulating hormone; LDL, low-density lipoprotein; LH, luteinizing hormone; TCHO, total cholesterol; NLR, neutrophil to lymphocyte ratio. 
fibroids can be treated conservatively. However, some uterine fibroids can grow rapidly and cause acute adverse events. Therefore, in patients with asymptomatic fibroids, it is important to predict fibroids' growth rate for followup and monitoring. This study constructed a predictive

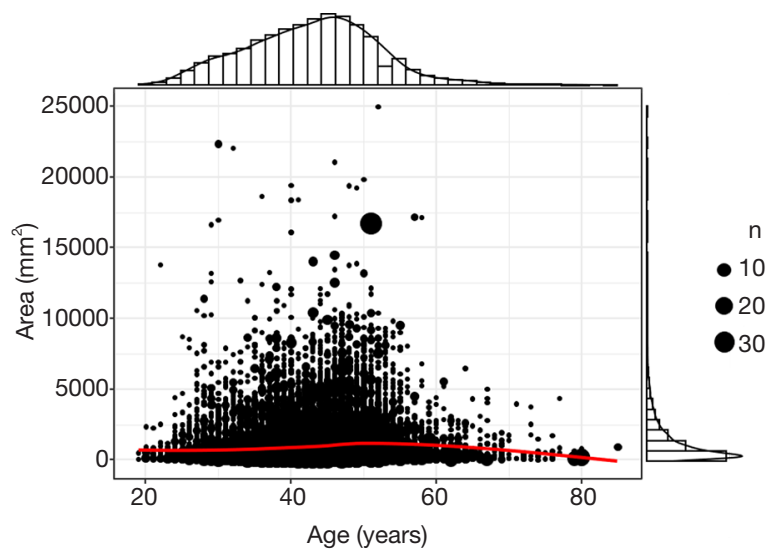

Figure 2 A risk prediction nomogram of significant uterine fibroid growth. model to screen patients with asymptomatic single-onset uterine fibroids for possible rapid fibroid growth. The predictive outcomes provide an early warning to increase the frequency of monitoring, thereby reducing acute uterine fibroid events.

A retrospective analysis of clinical data from patients with uterine fibroids was performed. Logistic regression models were constructed to predict patients who were likely to have rapidly growing uterine fibroids (growth ratio $>0.25 \mathrm{~mm}^{2} / \mathrm{d}$ ). A linear regression model was constructed to predict the uterine fibroid growth rate for patients with potential rapid fibroid growth. Figure 1 is a flowchart for the predictive model. Data regarding age and the average FSH levels, LH, NLR, LDL, and TCHO, were collected. The logistic regression model was used to predict the growth of fibroids. If the prediction is negative, the patient is considered to have a slow-growing uterine fibroid. If the result is positive, the uterine fibroids are considered to have a faster growth rate. Patients with positive predictions from the logistic model can predict the myoma growth rate in a linear regression model. The linear regression model
A

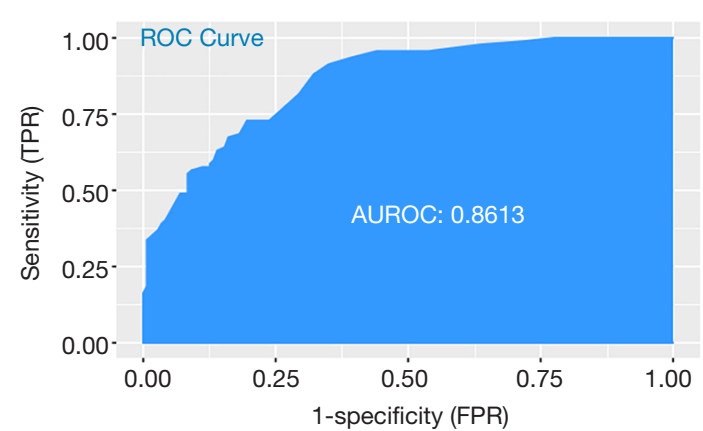

C

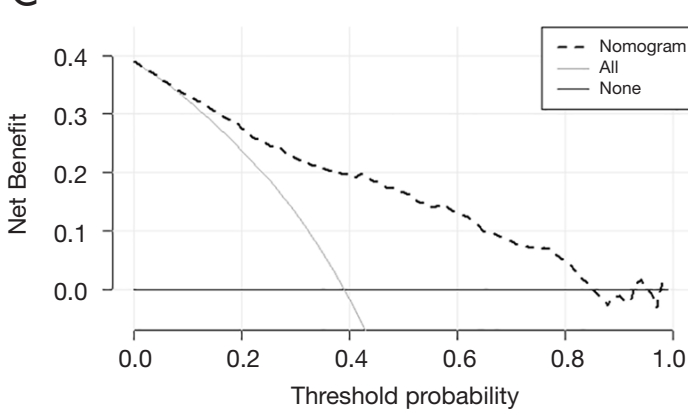

B
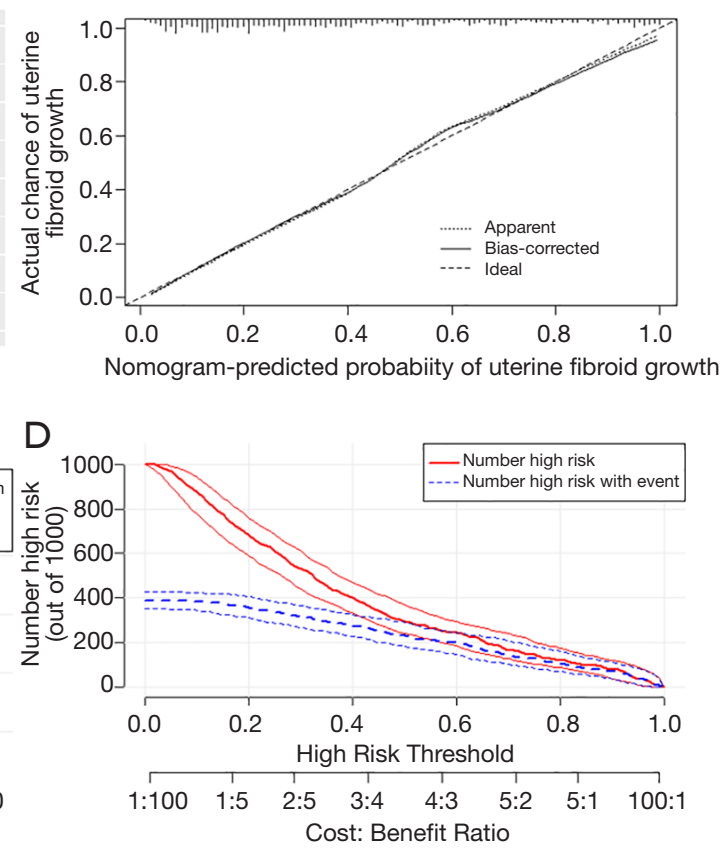

Figure 3 Evaluation of the predictive model for uterine fibroid growth. (A) The ROC curve of the logistics model in the test group predicting significant growth of uterine fibroids; (B) the calibration curve of the risk prediction nomogram predicting significant growth of uterine fibroids; (C) the decision analysis curve of the risk prediction model; (D) the clinical impact curve of the risk prediction model. TPR, true positive rate; FPR, false positive rate; ROC, receiver operating characteristic; AUROC, area under the receiver operating characteristic curve. 
Table 6 Linear regression prediction model for the uterine fibroid growth ratio

\begin{tabular}{lc}
\hline Factor & Regression coefficient \\
\hline FSH & 0.04 \\
HDL & -1.08 \\
LH & 0.04 \\
TCHO & 0.25 \\
TRIG & -0.14 \\
LMR & 0.04 \\
Intercept & -0.12 \\
\hline
\end{tabular}

$\mathrm{FSH}$, follicle-stimulating hormone; HDL, high-density lipoprotein; $\mathrm{LH}$, luteinizing hormone; TCHO, total cholesterol; TRIG, triglyceride; LMR, lymphocyte to monocyte ratio.

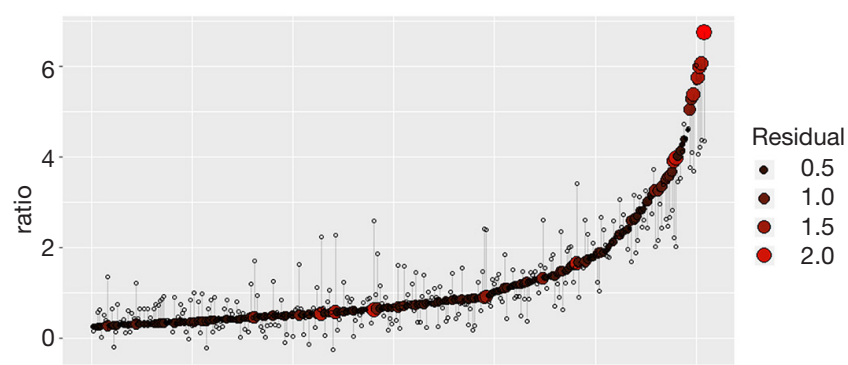

Figure 4 A linear regression model was used to predict the growth rate of the positive growth group. The red circle represents the actual growth ratio, and the color represents the residuals. The white circle above the red circle indicates that the residual value is positive, otherwise, the residual value is negative.

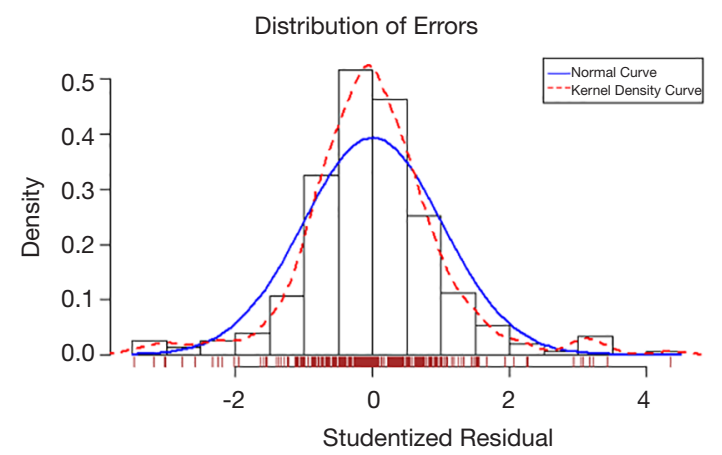

Figure 5 The studentized residual showed that the errors predicted by the linear regression model obeyed the normal distribution. requires the collection of FSH, HDL, LH, TCHO, TRIG, and LMR values to predict the uterine fibroids' growth rate. Physicians may recommend more frequent follow-up for patients with faster fibroid growth rates.

The uterine growth prediction model incorporated 6 factors, including age, FSH, LH, NLR, LDL, and TCHO. These 6 factors were included in the linear regression model to predict the uterine fibroid growth rate. FSH and LH are factors in both models, and these are currently recognized to have growth-promoting effects on uterine fibroids (18). Our model also suggested that these 2 factors positively affect the growth of uterine fibroids and growth rate. Other studies have suggested that estrogen may promote the growth of uterine fibroids (19). Growth hormone synergizes with estrogen to promote mitosis and fibroid growth. Researchers have also speculated that human placental lactogen synergizes with estrogen to promote fibroid growth (20). The current study found that the average estrogen concentration in the positive growth group was higher than that in the negative growth group. However, estrogen levels fluctuated widely, and no significant differences were found in the statistical analysis.

Age has a complex effect on the growth of uterus fibroids. Fibroids are rare in women younger than 20 years of age, but women aged 30-50 have a higher prevalence of uterine fibroids. After the age of 50 years, uterine fibroids may shrink (21). The age range in the logistic regression model was $20-55$ years. Beyond this range, the influence of age on uterine fibroids appeared uncertain. Before the age of 20 and after the age of 50, hormone levels are significantly lower than during the childbearing years. Therefore, the uterus grows the fastest during the reproductive years.

The three factors HDL, TCHO, and TRIG, which are common to both models, are related to lipid metabolism. HDL carries cholesterol in surrounding tissues converted to bile acids or excreted directly from the intestine through bile. It can effectively reduce the concentration of TCHO and TRIG. The relationship between lipid metabolism and uterine fibroid growth is currently unclear (22-24). Some studies believe that fat accumulation leads to chronic inflammation, which can promote the growth of uterine fibroids (25). In our model, HDL was negatively correlated with the growth rate of uterine fibroids.

There were some limitations to this study. First, the 
number of patients with uterine fibroids was relatively small, and the results require further validation with a larger study cohort. Second, the mean follow-up time in this study was 948 days, and a longer follow-up time would further verify these results.

In this investigation, two complementary models were constructed to identify potential rapid fibroid growth in patients with a single asymptomatic uterine fibroid episode.

\section{Acknowledgments}

The data processing involved in this study was powered by the Network and Information Technology Department of Huazhong University of Science and Technology Union Shenzhen Hospital.

Funding: This work was financed by grants from the National Natural Science Foundation of China (No. 81971341).

\section{Footnote}

Reporting Checklist: The authors have completed the TRIPOD reporting checklist. Available at http://dx.doi. org/10.21037/atm-20-4559

Data Sharing Statement: Available at http://dx.doi. org/10.21037/atm-20-4559

Conflicts of Interest: All authors have completed the ICMJE uniform disclosure form (available at http://dx.doi. org/10.21037/atm-20-4559). The other authors have no conflicts of interest to declare.

Ethical Statement: The authors are accountable for all aspects of the work in ensuring that questions related to the accuracy or integrity of any part of the work are appropriately investigated and resolved. The study was conducted in accordance with the Declaration of Helsinki (as revised in 2013) and was approved by the institutional research ethics committee of Huazhong University of Science and Technology Union Shenzhen Hospital and the accessed data were anonymized (ID: LW-2020-007). Because of the retrospective nature of the research, the requirement for informed consent was waived.

Open Access Statement: This is an Open Access article distributed in accordance with the Creative Commons Attribution-NonCommercial-NoDerivs 4.0 International
License (CC BY-NC-ND 4.0), which permits the noncommercial replication and distribution of the article with the strict proviso that no changes or edits are made and the original work is properly cited (including links to both the formal publication through the relevant DOI and the license). See: https://creativecommons.org/licenses/by-nc-nd/4.0/.

\section{References}

1. Stewart EA, Cookson C, Gandolfo R, et al. Epidemiology of uterine fibroids: a systematic review. BJOG

2017;124:1501-12.

2. Volkers NA, Hehenkamp WJK, Smit P, et al. Economic evaluation of uterine artery embolization versus hysterectomy in the treatment of symptomatic uterine fibroids: results from the randomized EMMY trial. J Vasc Interv Radiol 2008;19:1007-16.

3. Morgan DM, Kamdar NS, Swenson CW, et al. Nationwide trends in the utilization of and payments for hysterectomy in the United States among commercially insured women. Am J Obstet Gynecol 2018;218:425.e1-18.

4. Commandeur AE, Styer AK, Teixeira JM. Epidemiological and genetic clues for molecular mechanisms involved in uterine leiomyoma development and growth. Hum Reprod Update 2015;21:593-615.

5. Wong JY, Gold EB, Johnson WO, et al. Circulating Sex Hormones and Risk of Uterine Fibroids: Study of Women's Health Across the Nation (SWAN). J Clin Endocrinol Metab 2016;101:123-30.

6. Christopoulos G, Vlismas A, Salim R, et al. Fibroids that do not distort the uterine cavity and IVF success rates: an observational study using extensive matching criteria. BJOG 2017;124:615-21.

7. Gerges B, Mongelli M, Casikar I, et al. Three-dimensional transvaginal sonographic assessment of uterine volume as preoperative predictor of need to morcellate in women undergoing laparoscopic hysterectomy. Ultrasound Obstet Gynecol 2017;50:255-60.

8. Reis FM, Bloise E, Ortiga-Carvalho TM. Hormones and pathogenesis of uterine fibroids. Best Pract Res Clin Obstet Gynaecol 2016;34:13-24.

9. Sommer EM, Balkwill A, Reeves G, et al. Effects of obesity and hormone therapy on surgically-confirmed fibroids in postmenopausal women. Eur J Epidemiol 2015;30:493-9.

10. Palomba S, Affinito P, Di Carlo C, et al. Long-term administration of tibolone plus gonadotropin-releasing hormone agonist for the treatment of uterine leiomyomas: effectiveness and effects on vasomotor symptoms, bone 
mass, and lipid profiles. Fertil Steril 1999;72:889-95.

11. Sato F, Nishi M, Kudo R, et al. Body fat distribution and uterine leiomyomas. J Epidemiol 1998;8:176-80.

12. Wegienka G. Are uterine leiomyoma a consequence of a chronically inflammatory immune system? Med Hypotheses 2012;79:226-31.

13. Chen L. Overview of clinical prediction models. Ann Transl Med 2020;8:71.

14. Zhou ZR, Wang WW, Li Y, et al. In-depth mining of clinical data: the construction of clinical prediction model with R. Ann Transl Med 2019;7:796.

15. Rousson V, Zumbrunn T. Decision curve analysis revisited: overall net benefit, relationships to ROC curve analysis, and application to case-control studies. BMC Med Inform Decis Mak 2011;11:45.

16. Vickers AJ, Elkin EB. Decision curve analysis: a novel method for evaluating prediction models. Med Decis Making 2006;26:565-74.

17. Kerr KF, Brown MD, Zhu K, et al. Assessing the Clinical Impact of Risk Prediction Models With Decision Curves: Guidance for Correct Interpretation and Appropriate Use. J Clin Oncol 2016;34:2534-40.

18. Huirne JA, Lambalk CB. Gonadotropin-releasinghormone-receptor antagonists. Lancet 2001;358:1793-803.

19. Luo N, Guan Q, Zheng L, et al. Estrogen-mediated

Cite this article as: Li Q, Zhong J, Yi D, Deng G, Liu Z, Wang W. Assessing the risk of rapid fibroid growth in patients with asymptomatic solitary uterine myoma using a multivariate prediction model. Ann Transl Med 2021;9(5):370. doi: 10.21037/atm-20-4559 activation of fibroblasts and its effects on the fibroid cell proliferation. Transl Res 2014;163:232-41.

20. Zhang J, Sun Y, Liu Y, et al. Synergistic effects of androgen and estrogen on the mouse uterus and mammary gland. Oncol Rep 2004;12:709-16.

21. Pavone D, Clemenza S, Sorbi F, et al. Epidemiology and Risk Factors of Uterine Fibroids. Best Pract Res Clin Obstet Gynaecol 2018;46:3-11.

22. Zeybek B, Costantine M, Kilic GS, et al. Therapeutic Roles of Statins in Gynecology and Obstetrics: The Current Evidence. Reprod Sci 2018;25:802-17.

23. Vignini A, Sabbatinelli J, Clemente N, et al. Preperitoneal Fat Thicknesses, Lipid Profile, and Oxidative Status in Women With Uterine Fibroids. Reprod Sci 2017;24:1419-25.

24. Pejić S, Kasapovic J, Todorovic A, et al. Lipid peroxidation and antioxidant status in blood of patients with uterine myoma, endometrial polypus, hyperplastic and malignant endometrium. Biol Res 2006;39:619-29.

25. El Andaloussi A, Chaudhry Z, Al-Hendy A, et al. Uterine Fibroids: Bridging Genomic Defects and Chronic Inflammation. Semin Reprod Med 2017;35:494-8.

(English Language Editors: J. Teoh and J. Chapnick) 


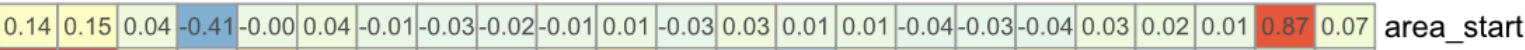

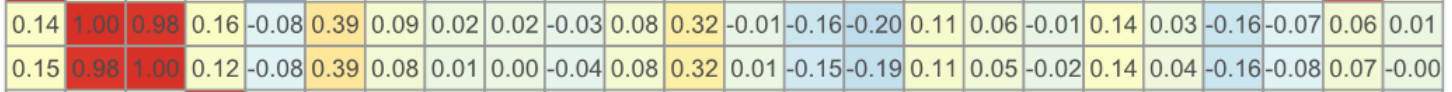
age_end $0.040 .16 \mid 0.12$ 0.010 .06

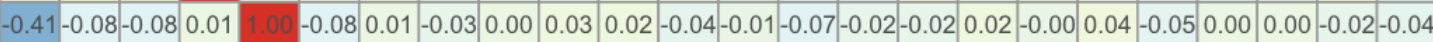

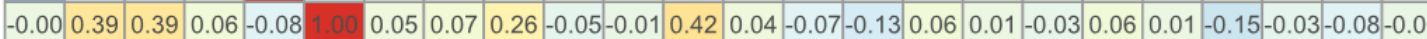

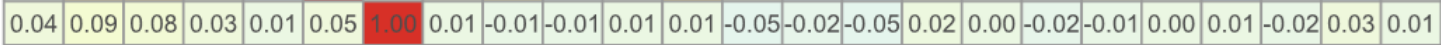
\begin{tabular}{|l|l|l|l|l|l|l|l|l|l|l|l|l|l|l|l|l|l|l|l|l|l|l|l}
-0.01 & 0.02 & 0.01 & -0.02 & -0.03 & 0.07 & 0.01 & 1.00 & -0.08 & -0.03 & 0.02 & 0.05 & 0.24 & 0.16 & 0.12 & 0.05 & 0.01 & 0.01 & 0.00 & -0.00 & -0.04 & -0.04 & -0.03 & 0.05 \\
\hline
\end{tabular}

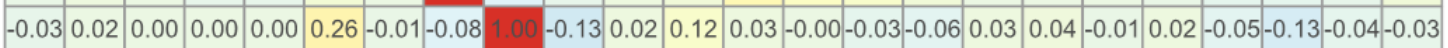
$-0.02-0.03-0.04-0.020 .03-0.05-0.01-0.03-0.13$ \begin{tabular}{ll|l|l|l|l|l|l|l|l|l|l}
-0.01 & 0.08 & 0.08 & 0.00 & 0.02 & -0.01 & 0.01 & 0.02 & 0.02 & 0.27
\end{tabular}

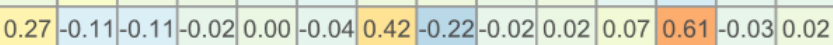
age_start diet

$-0.04$

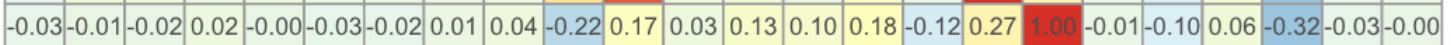

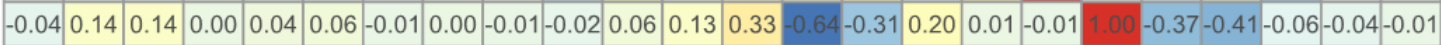

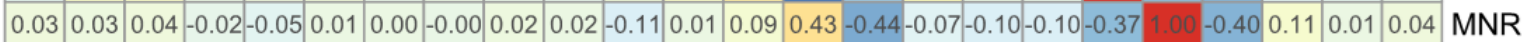

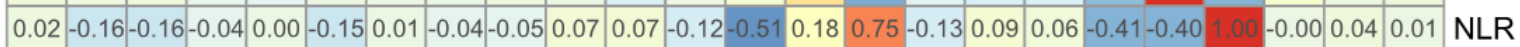
$0.01-0.07-0.08-0.020 .00-0.03-0.02-0.04-0.130 .61-0.55-0.12-0.14-0.01-0.10-0.05-0.33-0.32-0.060 .11-0.001 .00-0.020 .01$ HLR

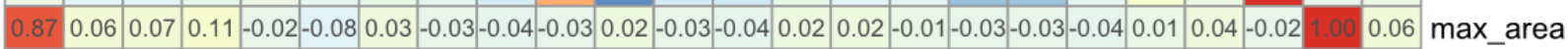

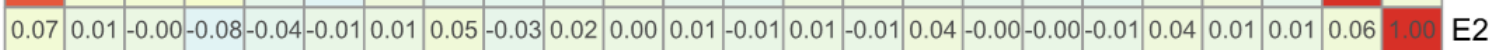

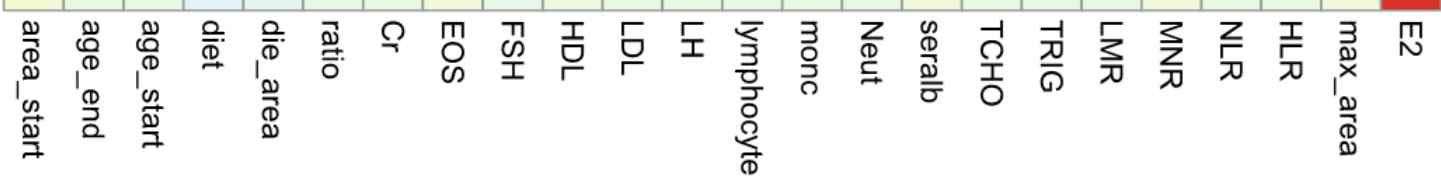

Figure S1 The correlation between the various clinically tested factors. The color and value represent the correlation coefficient. Cr, creatinine; EOS, eosinophil; FSH, follicle-stimulating hormone; HDL, high-density lipoprotein; LDL, low-density lipoprotein; LH, luteinizing hormone; Monc, monocytes; Neut, neutrophils; TCHO, total cholesterol; TRIG, triglyceride; LMR, lymphocyte to monocyte ratio; MNR, monocytes to neutrophil ratio; NLR, neutrophil to lymphocyte ratio; HLR, high-density lipoprotein to low-density lipoprotein ratio; E2, estradiol. 